\title{
The Planetary Spectroscopy Laboratory (PSL) at DLR in Berlin: transmission, reflectance and emissivity spectroscopy from UV to FIR
}

\author{
by A. Maturilli* and J.Helbert*
}

\author{
* German Aerospace Center (DLR), Institute of Planetary Research, Rutherfordstrasse 2, 12489, Berlin, \\ Germany, alessandro.maturilli@dlr.de, joern.helbert@dlr.de
}

\begin{abstract}
Since more than a decade the Planetary Emissivity Laboratory (PEL) at DLR in Berlin has provided spectral measurements of planetary analogues from the visible to the far-infrared range for comparison with remote sensing spacecraft/telescopic measurements of planetary surfaces. Bi-directional reflection, transmission and emission spectroscopy are the techniques we used to acquire spectral data of target materials. In 2007 we started investigating the effects of temperature on the emissivity spectra of planetary analogue materials.

In fall 2015 we performed a major upgrade to our laboratory set-up, adding a new spectrometer, three external sources, and new detectors and beamsplitters to further extend the spectral range of measurements that can be performed in the laboratory. To better reflect the new enhanced capabilities we renamed it to Planetary Spectroscopy Laboratory (PSL). PSL is a worldwide unique facility for the planetary science community, with a spectral range from UV $(0.2 \mu \mathrm{m})$ to the FIR $(200 \mu \mathrm{m}$ and above), and a range of sample temperature from $70 \mathrm{~K}$ to $1000 \mathrm{~K}$ under vacuum conditions. The purpose of this paper is to illustrate all of the possible measurements that can be performed at the Planetary Spectroscopy Laboratory (s.dlr.de/2siu).
\end{abstract}

\section{Introduction}

Spectroscopy is still the most accurate methodology to remotely study the surface composition of celestial bodies (and its evolution). For more than ten years the Planetary Emissivity Laboratory (PEL) of DLR in Berlin has provided spectral measurements of planetary analogues from the visible to the far-infrared range for comparison with remote sensing spacecraft/telescopic measurements of extra-terrestrial surfaces [1-5]. Reflection, transmission and emission spectroscopy are the techniques we used to acquire spectral data of target materials.

A recent major upgrade to our laboratory set-up added a new spectrometer, three external sources, optical units, new detectors and beamsplitters to further extend the spectral range of measurements that can be performed in the laboratory, as well as the temperature range that we can cover for the measurements. The purpose of this paper is to illustrate the very wide range of capabilities that the Planetary Spectroscopy Laboratory (PSL) can offer to the planetary and to the spectroscopic community.

\section{PSL Set-up description}

Two identical FTIR (Fourier Transform Infrared Spectrometers) are operating at PSL, in an air-conditioned room (Figure 1). The two spectrometers are both the same model, Bruker Vertex $80 \mathrm{~V}$ that can be evacuated to $\sim .1 \mathrm{mbar}$. One spectrometer is equipped with aluminium mirrors optimized for the UV, visible and near-IR, the second features goldcoated mirrors for the near to far IR spectral range. Using two identical instruments (apart from the internal mirrors) has some major benefits. The instruments can share the collection of detectors, beamsplitters, and optical accessories that we have in our equipment to cover a very wide spectral range, and this facilitates the cross-calibration between the two instruments. The instruments and the optical accessory units used are fully automatized and the data calibration and reduction are made with quality controlled software, developed following the DLR quality management rules. Table 1 list the amount of detectors that we have available at PSL and the spectral range in which those devices are used, Table 2 describes the associated beamsplitters we have in use at PSL.

Three external sources feature the PSL set-up: a deuterium lamp is used to cover the UV $(0.2$ to $0.5 \mu \mathrm{m})$ spectral range; a $24 \mathrm{~V}$, water cooled, Tungsten lamp has been added for measurements in the VIS (0.4 to $1.1 \mu \mathrm{m})$ spectral range; high power Globar lamp (24 V, water cooled) is used in the VNIR+TIR (1 to $16 \mu \mathrm{m})$ spectral range. Complimentary to the external ones, two internal sources from VIS to FIR complete the available offer. 


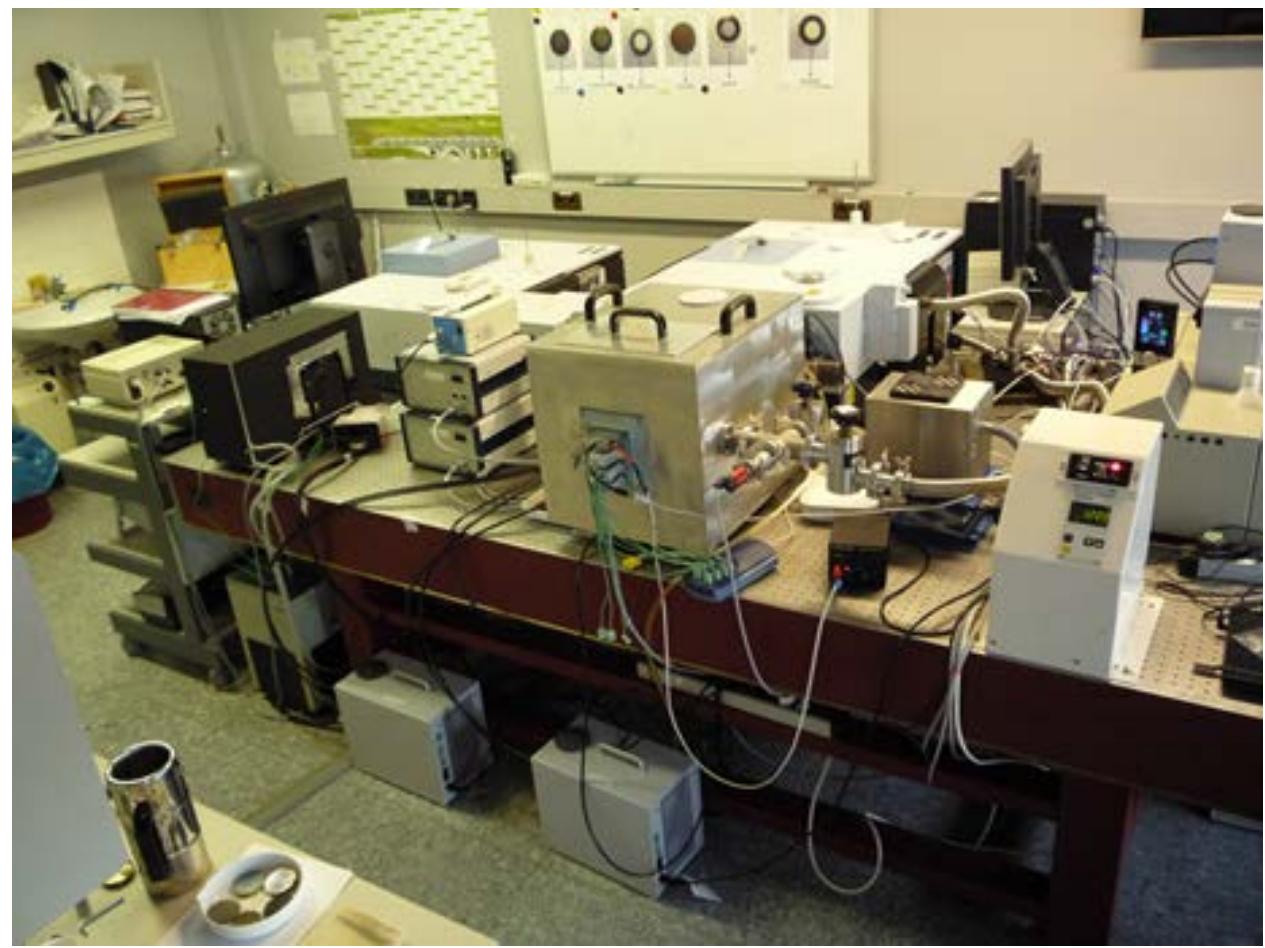

Fig. 1. The Laboratory set-up at the PSL

Table 1. Collection of detectors equipment available at PSL

\begin{tabular}{|l|l|l|}
\hline Detector & Spectral Range $(\mu \mathrm{m})$ & Operating T \\
\hline GaP Diode & $0.2-0.55$ & Room T \\
\hline 2x Silicon Diode & $0.4-1.1$ & Room T \\
\hline 2x InGaAs Diode & $0.7-2.5$ & Room T \\
\hline InSb & $0.78-5.4$ & Liquid $\mathrm{N}_{2}$ \\
\hline $2 \times \mathrm{MCT}$ & $0.8-16$ & Liquid $\mathrm{N}_{2}$ \\
\hline $\mathrm{MCT} / \mathrm{InSb} \mathrm{SW}$ & $1-16$ & Liquid $\mathrm{N}_{2}$ \\
\hline 2x DTGS/KBr & $0.8-40$ & Room T \\
\hline DTGS/CsI & $0.8-55$ & Room T \\
\hline DTGS/PE & $14-1000$ & Room T \\
\hline
\end{tabular}

Table 2. Collection of beamsplitters equipment available at PSL

\begin{tabular}{|l|l|}
\hline Beamsplitter & Spectral Range $(\mu \mathrm{m})$ \\
\hline $2 x$ UV/VIS/NIR CaF & \\
\hline $2 x$ Si on CaF 2 & $0.18-2.5$ \\
\hline 2x Ge on $\mathrm{KBr}$ (Wide) & $0.66-8.3$ \\
\hline Ge on KBr substrate & $1-25$ \\
\hline Multilayer & $1.2-25$ \\
\hline $50 \mu \mathrm{m}$ Mylar & $14.7-333$ \\
\hline & $181-666$ \\
\hline
\end{tabular}




\section{PSL Support Equipment}

Sample preparation, analysis tools, and experiment sub-systems are available in the facility. The full layout is shown in Figure 2. We have a collection of hundreds of rocks and minerals, several synthetic minerals, one Apollo 16 lunar sample, meteorites. This is complemented by our sample preparation and characterization lab operated by a dedicated technician. This includes two milling setups, two automated sieving setups with a wide range of sets of sieves, an ultrasonic cleaning unit, grinders, mortars, saw, balances, two stereo microscopes, two ovens (290 to $570 \mathrm{~K}$ and 300 to $3300 \mathrm{~K}$ ) for sample treatments, wet chemistry materials, and a pellets press (10 mm or $20 \mathrm{~mm}$ diameter). .Typical grain size separates produced for our spectral measurements are <25 $\mu \mathrm{m}, 25-63 \mu \mathrm{m}, 63-125 \mu \mathrm{m}, 125-250 \mu \mathrm{m}$; larger separates as well as slabs are produced if needed. For storing sensitive samples we have three large dry cabinets (moisture $<1 \%$ ) for sample storage as well as several small desiccators (moisture $<20 \%$ ). A purge gas generator for water and $\mathrm{CO}_{2}$ free air and a liquid-nitrogen tank complements the technical lab support.

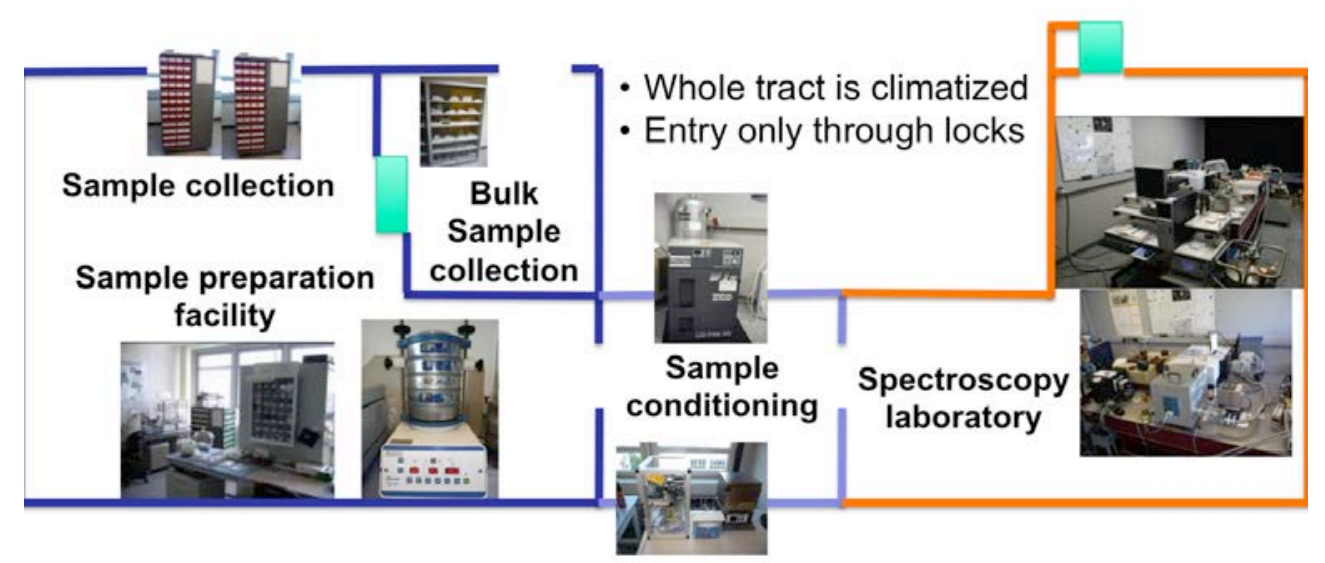

Fig. 2. The complete set-up equipment at the $P S L$

Building on the available infrastructure and the long heritage DLR is planning to create a Planetary Sample Analysis laboratory (PSA), which can be later extended to a full Sample Curation facility. PSA will focus on spectroscopy on the microscopic scale and geochemical analysis methods to study elemental composition and isotopic ratios in addition to mineralogy to derive information on the formation and evolution of planetary surface search for traces of organic materials and inclusions of water. To characterize and analyse the samples (including extra-terrestrial returned samples) we will add an Electron Microprobe Analyse (EMPA) for elemental analysis; a Laser ablated inductive coupled Plasma Mass Spectrometer for elemental and isotope analysis, and a Dual Source TXRF \& Grazing Incidence ED-XRF for mineralogical and structural analysis. The DLR PSA will be operated as a community facility (much like PSL), supporting the larger German and European sample analysis community

\section{Spectral measurement of sample emissivity}

External simulation chambers are attached to each of the FTIR spectrometers to measure the emissivity of solid samples. One chamber features a high efficiency induction system to heat samples under vacuum to temperatures from $320 \mathrm{~K}$ up to above $900 \mathrm{~K}$, while keeping the chamber at almost ambient temperature. A shutter allows separating the spectrometer from the external chamber. The advantages of a shutter separating the external chamber from the spectrometer are plentiful: samples can be heated at vacuum pressure lower than that available inside the spectrometer (vacuum in the spectrometer is limited by the air-bearing for the mirror movements); in case of outgassing, no harm is caused to the sensible optical parts inside the spectrometer; optical parts inside the spectrometer are separated from the hot sample environment, hence they are not heated, also their optical properties are not changing during a day of measurements; spectrometer can be vented and optical units exchanged while the samples in the external chamber remains in vacuum. An optical window (vacuum tight) can be mounted at the entrance of the emissivity chamber to allow keeping the external chamber at $\geq$ ambient pressure, under purged air or inert gases. The sample cups are made of stainless steel and have elevated rims enclosing the samples heating it from all sides, effectively supressing thermal gradients within the samples. A sample carousel driven by a highly precise stepper motor allows measuring several consecutive samples without breaking the vacuum. A large number of temperature sensors in the emissivity chamber are allocated to measure the sample/cup temperature as well as monitoring the equipment and chamber temperatures. A webcam is mounted in the emissivity chamber to monitor the heated sample and its vicinity; it is usually used to take 
pictures of the sample at each measuring temperature, but can also be used to obtain a video-recording of an experiement. The latter can add precious information on the thermal processes occurring to the heated materials in the emissivity chamber.

Each sample is poured in a stainless steel cup to a $3 \mathrm{~mm}$ thick uniform layer; the cup is placed on an induction pancake-coil. Induction heating is used to heat up the cup and thereby the samples contained. By using the induction properties to heat the cups, we do not set the heater temperature, but the intensity of the current that we send to the induction coil. The whole cup (made of steel) is heated uniformly by the induction process; therefore our samples are heated from below as well as from the side (rims) of the cup, and are not heated/illuminated from above. A thermopile temperature sensor (whose head is a long, thin wire of $0.2 \mathrm{~mm}$ ) is put in contact with the emitting surface, to read the sample temperature. Blast furnace slag, poured in a stainless steel cup and under exactly the same geometric configuration as the samples, is heated to sample temperature and is used as calibration blackbody. The calibrated sample emissivity is then calculated by dividing the sample radiance to the blackbody radiance (measured at the same temperature), and then multiplying this resulting spectrum for the emissivity spectrum of the blackbody material. The emissivity curve of the slag has been retrieved by measuring the blast furnace slag vs. a commercial blackbody, painted with a black and well characterized coating (Acktar Fractal Black). Figure 3 shows an example of emissivity measurement taken in vacuum for a quartz sample $(125-250 \mu \mathrm{m}$ grain size range) at various high temperatures. It can be noted that for the emissivity spectrum for this sample taken at $666^{\circ} \mathrm{C}$ surface temperature shows the spectral effects of the $\alpha$ - to $\beta$ - transition typical for the tetrahedral molecule of quartz (a continuous framework of SiO4). In fact, the roomtemperature form of quartz, $\alpha$-quartz, undergoes a reversible change in the crystal structure at $573^{\circ} \mathrm{C}$ to form the $\beta$ quartz.

In a recent upgrade we added capabilities to measure emissivity at wavelengths as low as $0.7 \mu \mathrm{m}$, to specifically address the needs of the Venus science community. This upgrade was funded by the European Commission European Union's Horizon 2020 research and innovation programme under grant agreement No 654208 The permanent cloud cover of Venus prohibits observation of the surface with traditional imaging techniques over most of the visible spectral range. Venus' CO2 atmosphere is only transparent in small spectral windows near $1 \mu \mathrm{m}$. However, using stainless steel cups to heat samples to very high temperatures has a disadvantage in the visible spectral range. As the emissivity of steel increases significantly in the $1 \mu \mathrm{m}$ region at this high temperature the steel cup is visibly glowing and the emitted radiation from the sample cup can exceed the the sample emitted energy measured in the visible spectral range. To bypass this difficulty, ceramic cups enclosing a stainless steel disc are used to extend the high temperature spectroscopy capabilities of PSL to start at 700nm instead of $1000 \mathrm{~nm}$.

A second chamber (purged with dry air and water cooled to $270 \mathrm{~K}$ or below) is attached to the second spectrometer for emissivity measurements of samples with surface temperature from 290 to $420 \mathrm{~K}$. This second emissivity chamber is well suited for emissivity measurements of samples at temperatures close to room. It is covered with high emissivity black paint, also its walls reflect almost zero signal, while at the same time the cooled black walls emit very low signal.

Standard spectral resolution used for emissivity measurements is $4 \mathrm{~cm}^{-1}$ and the spot size is $49 \mathrm{~mm}$ for both emissivity set-ups. 


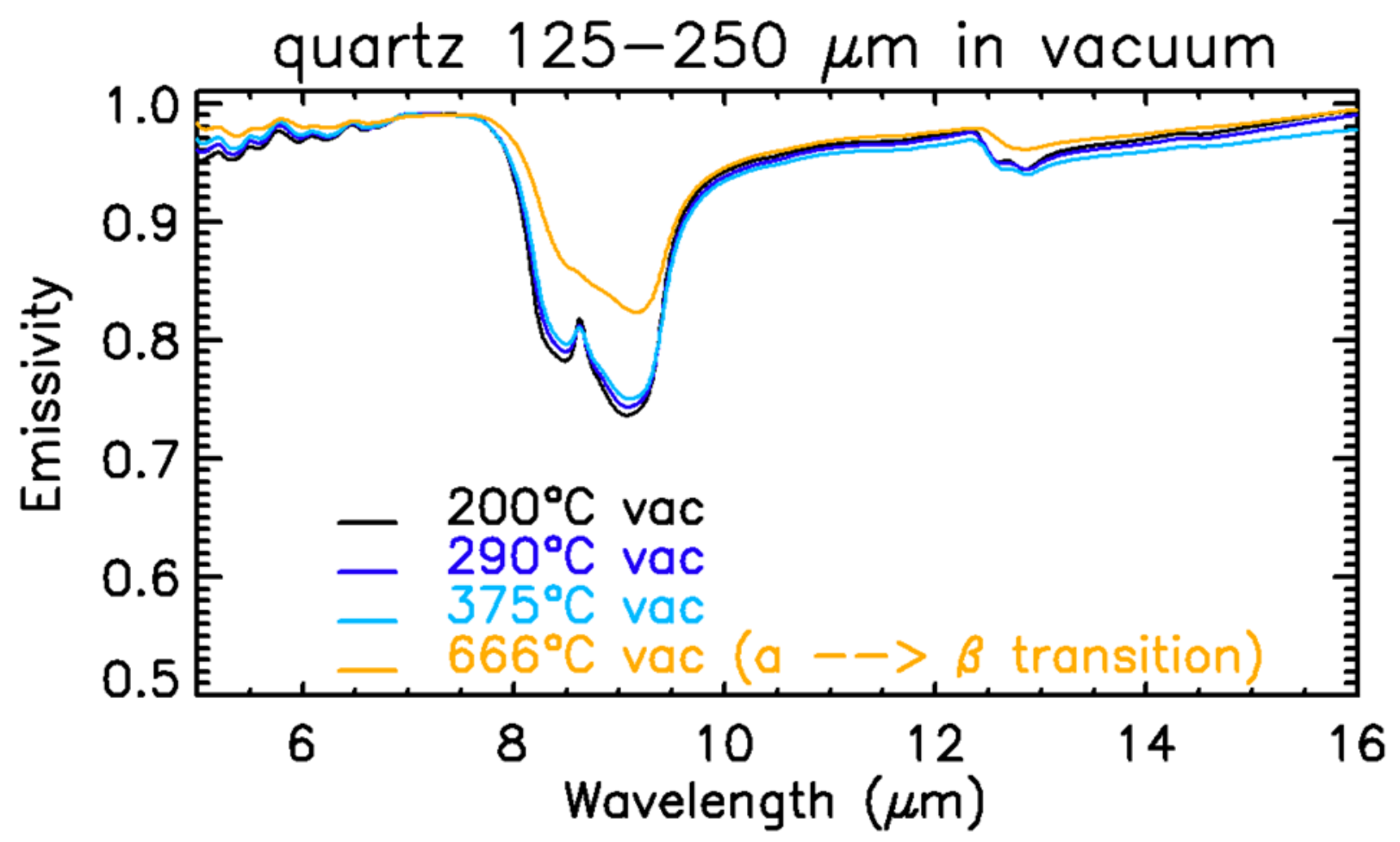

Fig. 3. Emissivity spectra of a quartz sample in vacuum at increasing temperatures

\section{Spectral measurement of sample reflectance}

With the Bruker A513 accessory on both Vertex 80V spectrometers, we measure bi-directional reflectance of samples, with variable incidence and emission angles between $13^{\circ}$ and $85^{\circ}$. The viewing cone of the A513 reflectance accessory has an aperture of $17^{\circ}$, small enough to define our measurements as bi-directional. Samples can be measured at room temperature and currently to $170 \mathrm{~K}$ using a test setup cooled by liquid nitrogen inside the spectrometer sample chamber. A compact low-temperature reflectance chamber for FT-spectroscopy experiments at the PSL is currently under development [7]. The expected cryogenic temperature to reach is approximately within the range of $70 \mathrm{~K}$ $-100 \mathrm{~K}$. We recently added two integrating spheres (one with gold coated surfaces, the other with PTFE coating) for hemispherical reflectance measurements. Reflectance measurements are calibrated by comparing with spectroscopic measurements of well characterised references. Standard spectral resolution used is $4 \mathrm{~cm}^{-1}$ and spot size is $2 \mathrm{~mm}$ for reflectance acquisitions. We measure bi-directional and hemispherical reflectance under purging or vacuum conditions, covering the 0.2 to above $200 \mu \mathrm{m}$ spectral range. Figure 4 shows an example of bi-directional reflectance measurement that we can perform on a very wide spectral range with our instruments at PSL. PlanetX is a mixture of minerals in the 0$25 \mu \mathrm{m}$ grain size range, developed in our laboratory to simulate a Mercury soil based on a anorthositic, low iron composition.
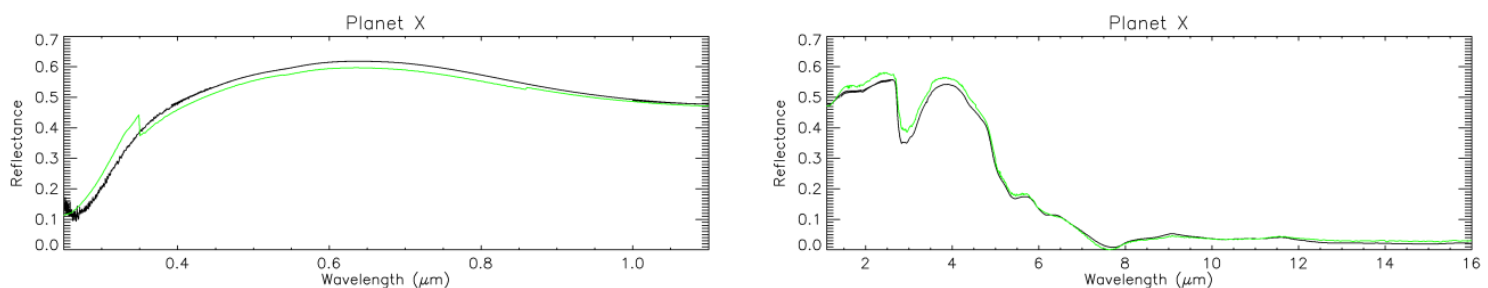

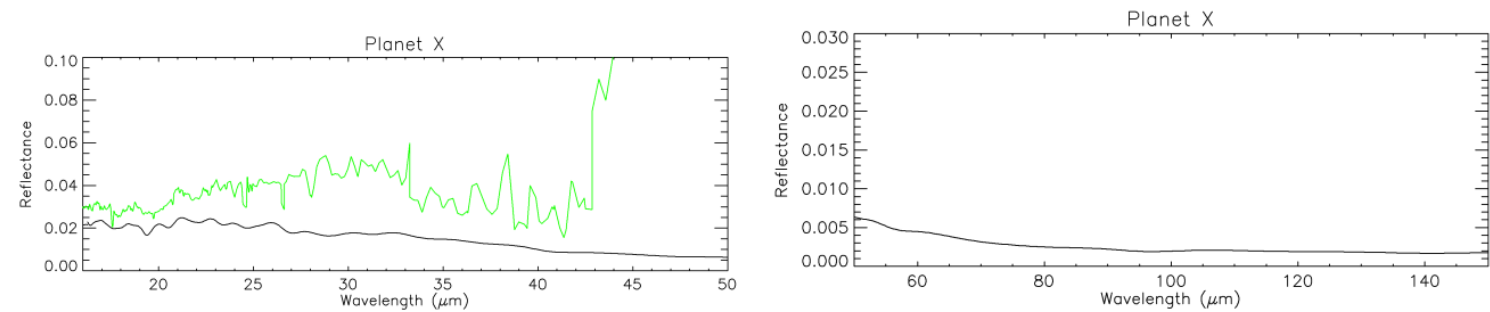

Fig. 4. Bi-directional reflectance spectrum of the PlanetX sample measured at the PSL in the whole spectral range from UV to the FIR $(0.2-150 \mu \mathrm{m})$

\section{Spectral measurement of sample transmittance}

The Bruker A480 parallel beam accessory mounted on the Vertex $80 \mathrm{~V}$ allows us to measure transmission of thin slabs, optical filters, optical windows, pellets, etc, in the complete spectral range from UV to FIR avoiding refraction, typical in this kind of measurements. Especially in the case of thick and highly refractive samples, refraction causes a shift of the IR-focus towards the detector, which is associated with an error in the transmittance values.

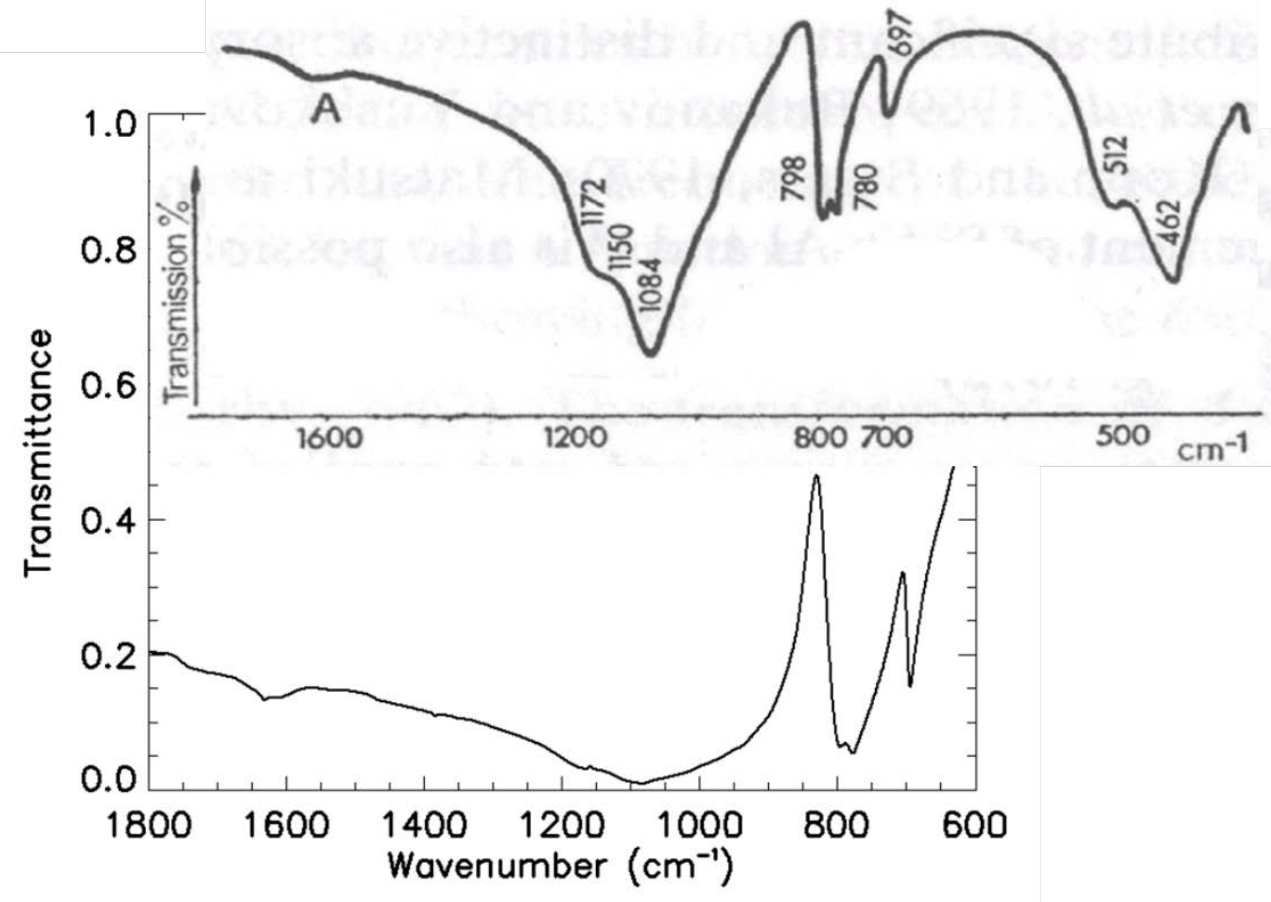

Fig. 5. Transmittance spectrum of a pellet made of $1 \%$ quartz $(0-25 \mu \mathrm{m})+99 \% \mathrm{KBr}$ (lower panel) compared with quartz transmittance spectrum taken from [8]

For oblique incidence, refraction gives rise to an additional error caused by a lateral beam shift. These inaccuracies can be overcome by measuring the sample in a parallel IR-beam with perpendicular incidence, as is realized in the parallel beam transmission accessory A480. Figure 5 shows the comparison between a sample measured in transmittance with the equipment available at PSL and a transmittance spectrum of the same specimen taken from [8]. Our sample for transmittance measurements was prepared by mixing together finely powdered $99 \% \mathrm{KBr}$ (in weight) and $1 \%$ quartz powder. The mixture was then pressed in vacuum with a Graseby Specac Hydraulic Press at 10 ton for 10 minutes. The transmittance spectrum of obtained pellet could then be measured in vacuum in one of our Bruker Vertex $80 \mathrm{~V}$ spectrometer. 


\section{Conclusion}

The Planetary Spectroscopy Laboratory (PSL) is constantly improving to provide the planetary and spectroscopic community with reflectance, transmission and emissivity measurements highly complementary to existing spectral databases, under vacuum, that cover the whole spectral range from UV $(0.2 \mu \mathrm{m})$ to the FIR $(200 \mu \mathrm{m}$ and above), and for sample temperature from $70 \mathrm{~K}$ to $1000 \mathrm{~K}$.

PSL is a Trans-national access (TA) facility supported by the European Union within the EuroPlanet Research Infrastructure framework for the next two years. In this period once per year a call for proposals will be issued for investigations using PSL.

PSL is also member of the NASA SSERVI Team TREX [9] providing spectral measurements of fine particle samples (https://trex.psi.edu/).

\section{REFERENCES}

[1] Maturilli, A. and Helbert, J, Emissivity measurements of analogue materials for the interpretation of data from PFS on Mars Express and MERTIS on Bepi-Colombo. Planetary and Space Science, Vol. 54, pp. 1057-1064, 2006.

[2] Maturilli, A., Helbert, J., and Moroz L., The Berlin Emissivity Database (BED), Planetary and Space Science, Vol. 56, pp. 420-425, 2008; spectral library now available at http://figshare.com/articles/BED_Emissivity_Spectral_Library/1536469.

[3] Helbert, J. and Maturilli, A, The emissivity of a fine-grained labradorite sample at typical Mercury dayside temperatures, Earth and Planetary Science Letters, Vol. 285(3), pp. 347-354, 2009.

[4] Maturilli A, Helbert J., Characterization, testing, calibration, and validation of the Berlin emissivity database, Journal of Applied Remote Sensing, 2014.

[5] Maturilli A, Helbert J, St. John J.M., Head III J.W., Vaughan W.M., D’Amore M., Gottschalk M., Ferrari S., Komatiites as Mercury Surface Analogues: Spectral Measurements at PEL, Earth and Planetary Science Letters, Vol. 398, pp. 58-65, 2014.

[6] Helbert, J., Maturilli, A., Dyar, M. D., Ferrari, S., Müller, N., Smrekar, S., Orbital Spectroscopy oft he Surface of Venus, Lunar and Planetary Science Conference, Abstract\# 1219, The Woodlands (Texas, USA), 2018.

[7] Rosas Ortiz, Y. M., Helbert, J., Maturilli, A., Lehman, M., A compact Planetary Simulation Chamber fort he Characterization oft he Bi-directional Reflectance of Asteroid, Cometary and Solar System Small Bodies (SSSB) analogues at Low-Temperature Environments, Lunar and Planetary Science Conference, Abstract\# 1883, The Woodlands (Texas, USA), 2018.

[8] Farmer, V. C., The Infrared Spectra of Minerals, Mineralogical Society Monograph 4, pp.1-539.

[9] Lane, M. D., Allain, J. P., Cahill, K. S., Clark, R. N., Cloutis, E. A., Dyar, M. D., Helbert, J., Hendrix, A. R., Holsclaw, G., Osterloo, M., Pearson, N., Savin, D. W., and the TREX team, Toolbox for Research and Exploration (TREX): The Fine-Particle Spectral Library, Lunar and Planetary Science Conference, Abstract\# 1098, The Woodlands (Texas, USA), 2018. 